\title{
WHEN WILL THE STANLEY DEPTH INCREASE?
}

\author{
YI-HUANG SHEN \\ (Communicated by Irena Peeva)
}

\begin{abstract}
Let $I \subset S=\mathbb{K},\left[x_{1}, \ldots, x_{n}\right]$ be an ideal generated by squarefree monomials of degree $\geq d$. If the number of degree $d$ minimal generating monomials is $\mu_{d}(I) \leq \min \left(\left(\begin{array}{c}n \\ d+1\end{array}\right), \sum_{j=1}^{n-d}\left(\begin{array}{c}2 j-1 \\ j\end{array}\right)\right)$, then the Stanley depth $\operatorname{sdepth}_{S}(I) \geq d+1$.
\end{abstract}

\section{INTRODUCTION}

Throughout this paper, let $\mathbb{K}$ be a field and $S=\mathbb{K}\left[x_{1}, \ldots, x_{n}\right]$ a polynomial ring in $n$ variables over $\mathbb{K}$. The ring $S$ has a natural $\mathbb{Z}^{n}$-grading. If $M$ is a finitely generated $\mathbb{Z}^{n}$-graded $S$-module, a Stanley decomposition of $M$ is a finite direct sum decomposition

$$
\mathcal{P}: M=\bigoplus_{i=1}^{m} u_{i} \mathbb{K}\left[Z_{i}\right]
$$

of $M$ as a $\mathbb{Z}^{n}$-graded $\mathbb{K}$-vector space, where each $u_{i} \in M$ is homogeneous and $Z_{i} \subset\left\{x_{1}, \ldots, x_{n}\right\}$. Here, $u_{i} \mathbb{K}\left[Z_{i}\right]$ is considered as a free $\mathbb{K}\left[Z_{i}\right]$-submodule of $M$. The Stanley depth of this decomposition is $\operatorname{sdepth}(\mathcal{P})=\min \left\{\left|Z_{i}\right|: 1 \leq i \leq m\right\}$, and the Stanley depth of the module $M$ is

$$
\operatorname{sdepth}(M):=\max \{\operatorname{sdepth}(\mathcal{P}): \mathcal{P} \text { is a Stanley decomposition of } M\} .
$$

If we consider isomorphism instead of equality in the previous Stanley decomposition $\mathcal{P}$, we will land up in the notion of a Hilbert depth, which is the main topic of BKU10.

The driving force for investigating the Stanley depth of a finitely generated $\mathbb{Z}^{n}$ graded module $M$ is the conjecture raised by Stanley [Sta82, which says

$$
\operatorname{sdepth}(M) \geq \operatorname{depth}(M) .
$$

This conjecture will imply ([HJY08, 4.5]) that Cohen-Macaulay simplicial complexes are partitionable, which was separately conjectured by Garsia Gar80, 5.2] and Stanley [Sta79, p. 149].

To have an insight into the properties of Stanley depth, one lacks the many powerful tools as those for the normal algebraic depth. Deciding the Stanley depth of interesting modules is already a headache for researchers. Currently, the Stanley

Received by the editors October 18, 2011.

2010 Mathematics Subject Classification. Primary 05E45, 05E40, 06A07; Secondary 13C13, $05 \mathrm{C} 70$.

Key words and phrases. Stanley depth, squarefree monomial ideal.

This work was supported by the National Natural Science Foundation of China (11201445) and the Fundamental Research Funds for the Central Universities (WK0010000017). 
depth is known only for a very narrow scope of modules, the overwhelming majority of which has equality in the Stanley conjecture $(\dagger)$.

The paper HVZ09] by Herzog, Vladoiu and Zheng was a breakthrough along this line. Their method attacks the problem of computing the Stanley depth $\operatorname{sdepth}(I / J)$ for monomial ideals $J \subset I$ in $S$. This method, though not a panacea, contributes fundamentally to the knowledge of Stanley decompositions from both theoretical and computational perspectives. For instance, based on this method, Biró et al. [BHK $\left.{ }^{+} 10\right]$ can show that $\operatorname{sdepth}_{S}\left(\left\langle x_{1}, \ldots, x_{n}\right\rangle\right)=\left\lceil\frac{n}{2}\right\rceil$. Notice that $\operatorname{depth}_{S}\left(\left\langle x_{1}, \ldots, x_{n}\right\rangle\right)=1$. Other nontrivial computations and estimates can be found in, for instance, KY09, Oka11, She09, and their references.

Throughout this paper, $I$ will be a monomial ideal in $S$, generated by squarefree monomials of degree $\geq d$. The task of the current paper is to investigate when $\operatorname{sdepth}(I) \geq d+1$. Our main result is the following theorem.

Theorem 1.1. Let $I \subset S=\mathbb{K}\left[x_{1}, \ldots, x_{n}\right]$ be an ideal generated by squarefree monomials of degree $\geq d$. If the number of degree $d$ minimal generating monomials

$$
\mu_{d}(I) \leq \min \left(\left(\begin{array}{c}
n \\
d+1
\end{array}\right), \sum_{j=1}^{n-d}\left(\begin{array}{c}
2 j-1 \\
j
\end{array}\right)\right)
$$

then the Stanley depth $\operatorname{sdepth}_{S}(I) \geq d+1$.

Let us finish this introduction by going over the structure of this paper. In section 2, we will go over Herzog, Vladoiu and Zheng's method for computing the Stanley depth of monomial ideals. We will tailor it to the squarefree case and prove a special case of the main theorem. In section 3, we will inspect several combinatorial constructions, which are essential for deciding when the Stanley depth will increase. In the final section, we will complete the proof and provide additional remarks and questions.

\section{Herzog, Vladoiu and Zheng's method}

By convention, we denote the set $\{1,2, \ldots, n\}$ by $[n]$. For the squarefree monomial ideal $I$, consider the associated set

$$
P_{I}:=\left\{\left\{i_{1}, \ldots, i_{m}\right\} \subset[n]: x_{i_{1}} \cdots x_{i_{m}} \in I, 1 \leq m \leq n\right\} .
$$

This is a partially ordered set (poset) with respect to inclusion. When $A, B \in P_{I}$, the interval $[A, B]$ is the set $\left\{C \in P_{I}: A \subset C \subset B\right\}$. Herzog, Vladoiu and Zheng's method [HVZ09, 2.5] for squarefree monomial ideals can easily be checked to be equivalent to the following characterization:

Lemma 2.1. Let $k$ be a positive integer. Then $\operatorname{sdepth}(I) \geq k$ if and only if $P_{I}$ has a disjoint partition $\mathcal{P}: P_{I}=\bigcup_{i=1}^{l}\left[A_{i}, B_{i}\right]$ such that the cardinalities $\left|B_{i}\right| \geq k$, $1 \leq i \leq l$.

This can be further simplified. Consider the reduced associated poset

$$
P_{I}^{k}:=\left\{\left\{i_{1}, \ldots, i_{m}\right\} \in P_{I}: m \leq k\right\} .
$$

It is partitionable if $P_{I}^{k}$ has a disjoint partition $\mathcal{P}: P_{I}^{k}=\bigcup_{i=1}^{l}\left[A_{i}, B_{i}\right]$ such that the cardinalities $\left|B_{i}\right|=k, 1 \leq i \leq l$. Thus, the previous lemma is equivalent to saying

$$
\operatorname{sdepth}(I) \geq k \Leftrightarrow P_{I}^{k} \text { is partitionable. }
$$


It follows that if $I$ is generated by squarefree monomials of degree $\geq d$, then $\operatorname{sdepth}(I) \geq d$. Meanwhile, we also have $\operatorname{depth}(I) \geq d$ in this case; see HVZ09, 1.3, $3.1]$.

Remark 2.2. Let $I$ be an $S$-ideal generated by squarefree monomials of degree $\geq d$ and $I_{d}$ the subideal generated by the degree $d$ generators of $I$. Since $P_{I}^{d+1}$ differs from $P_{I_{d}}^{d+1}$ only in the degree $d+1$ part, $\operatorname{sdepth}(I) \geq d+1$ if and only if $\operatorname{sdepth}\left(I_{d}\right) \geq d+1$. Using the observation $\operatorname{sdepth}(I), \operatorname{sdepth}\left(I_{d}\right) \geq d$, this is equivalent to saying that $\operatorname{sdepth}(I)=d$ if and only if $\operatorname{sdepth}\left(I_{d}\right)=d$. Thus, in the following, we may assume that $I=\left\langle I_{d}\right\rangle$; we will say $I$ is pure of degree $d$ in this case.

Remark 2.3. Let $I \subset J$ be two $S$-ideals which are generated by squarefree monomials of degree $d$. If $\operatorname{sdepth}(J) \geq d+1$, then $P_{J}^{d+1}$ is partitionable by $(\ddagger)$. The restriction of such a partition to $P_{I}^{d+1}$ shows that $P_{I}^{d+1}$ is also partitionable. Thus, $\operatorname{sdepth}(I) \geq d+1$. Notice that, in general, we cannot compare $\operatorname{sdepth}(I)$ with $\operatorname{sdepth}(J)$ even if there exists containment between $I$ and $J$. For instance, for the three squarefree monomial ideals $I_{1}:=\langle 1\rangle \supset I_{2}:=\left\langle x_{1}, x_{2}\right\rangle \supset I_{3}:=\left\langle x_{1}\right\rangle$ in $S=\mathbb{K}\left[x_{1}, x_{2}\right]$, we will have $\operatorname{sdepth}\left(I_{1}\right)=\operatorname{sdepth}\left(I_{3}\right)=2>\operatorname{sdepth}\left(I_{2}\right)=1$.

Corollary 2.4. Suppose $I$ is generated by squarefree monomials of degree $\geq d$ and $\operatorname{sdepth}(I) \geq d+1$. Then the number of degree $d$ minimal generators is $\mu_{d}(I) \leq$ $\left(\begin{array}{c}n \\ d+1\end{array}\right)$.

Proof. Since $\operatorname{sdepth}(I) \geq d+1, P_{I}^{d+1}$ is partitionable and has a partition $P_{I}^{d+1}=$ $\bigcup_{i=1}^{l}\left[A_{i}, B_{i}\right]$ with $\left|B_{i}\right|=d+1$. Now $\left(\begin{array}{c}n \\ d+1\end{array}\right) \geq\left|\left\{B_{i}: 1 \leq i \leq l\right\}\right| \geq\left|\left\{B_{i}:\left|A_{i}\right|=d\right\}\right|=$ $\left|\left\{A_{i}:\left|A_{i}\right|=d\right\}\right|=\mu_{d}(I)$.

When $n \geq 2 d+1$, we have $\mu_{d}(I) \leq\left(\begin{array}{l}n \\ d\end{array}\right) \leq\left(\begin{array}{c}n \\ d+1\end{array}\right)$. Thus Corollary 2.4 does not provide much information in this case. However, we have

Proposition 2.5. Suppose $n \geq 2 d+1$ and $I$ is generated by squarefree monomials of degree $\geq d$. Then $\operatorname{sdepth}(I) \geq d+1$.

Proof. Recall that the squarefree Veronese ideal $I_{n, d}$ is the ideal generated by all degree $d$ squarefree monomials of $S=\mathbb{K}\left[x_{1}, \ldots, x_{n}\right]$. It follows from [KSSY11, 1.1] that $\operatorname{sdepth}\left(I_{n, d}\right) \geq d+1$. Now, we use Remarks 2.2 and 2.3

Inspired by the proof of Proposition 2.5, we raise the following conjecture on the Stanley depth of squarefree monomial ideals:

Conjecture 2.6. If $I \subset S=\mathbb{K}\left[x_{1}, \ldots, x_{n}\right]$ is an ideal generated by squarefree monomials of degree $\geq d$, then

$$
\operatorname{sdepth}_{S}(I) \geq d+\left\lfloor\left(\begin{array}{c}
n \\
d+1
\end{array}\right) /\left(\begin{array}{l}
n \\
d
\end{array}\right)\right\rfloor .
$$

Thanks to $[\mathrm{KSSY11}, 2.2]$, we know $\operatorname{sdepth}\left(I_{n, d}\right) \leq d+\left\lfloor\left(\begin{array}{c}n \\ d+1\end{array}\right) /\left(\begin{array}{c}n \\ d\end{array}\right)\right\rfloor$. Hence this conjecture is stronger than the special case [KSSY11, 2.4], which is also separately conjectured by Cimpoeaş in [Cim09, 1.6]. 


\section{THE ASSOCIATED PURE COMPLEX}

Let $k$ be a positive integer. By [BH93, 4.2.6], any integer $x \geq 1$ can be written uniquely in the form

$$
x=\left(\begin{array}{c}
a_{k} \\
k
\end{array}\right)+\left(\begin{array}{c}
a_{k-1} \\
k-1
\end{array}\right)+\cdots+\left(\begin{array}{c}
a_{i} \\
i
\end{array}\right)
$$

such that $a_{k}>\cdots>a_{i} \geq i>0$. The above sum is called the $k$-th Macaulay representation of $x$. For any integer $j, 1 \leq j<i$, we set $a_{j}=j-1$. We shall call $a_{k}, a_{k-1}, \ldots, a_{1}$ the $k$-th Macaulay coefficients of $x$. They have the following nice property.

Lemma 3.1 ([BH93, 4.2.7]). Let $a_{k}, \ldots, a_{1}$, respectively $a_{k}^{\prime}, \ldots, a_{1}^{\prime}$, be the $k$-th Macaulay coefficients of $x$, respectively $x^{\prime}$. Then $x>x^{\prime}$ if and only if

$$
\left(a_{k}, \ldots, a_{1}\right)>\left(a_{k}^{\prime}, \ldots, a_{1}^{\prime}\right)
$$

in lexicographical order.

Now, let $\delta:=n-d$ be the difference of degrees and write $\xi_{\delta}:=\sum_{j=1}^{\delta}\left(\begin{array}{c}2 j-1 \\ j\end{array}\right)$. In Theorem 1.1 we need to compare the integer $\xi_{n-d}$ with $\left(\begin{array}{c}n \\ d+1\end{array}\right)$.

Lemma 3.2. Let $1 \leq \delta<n$. Then

$$
\min \left(\xi_{\delta},\left(\begin{array}{c}
n \\
\delta-1
\end{array}\right)\right)= \begin{cases}\xi_{\delta} & \text { if } n \geq 2 \delta \\
\left(\begin{array}{c}
n \\
\delta-1
\end{array}\right) & \text { if } n \leq 2 \delta-1 .\end{cases}
$$

Proof. The cases when $\delta=1$ and 2 can be verified directly. Thus, we assume that $\delta \geq 3$. Note that the $(\delta-1)$-th Macaulay coefficients of $\left(\begin{array}{c}n \\ \delta-1\end{array}\right)$ are $n, \delta-3, \delta-$ $4, \ldots, 1,0$. Meanwhile, the $(\delta-1)$-th Macaulay coefficients of $\xi_{\delta}$ are $2 \delta-1,2 \delta-$ $3, \ldots, 7,5,4$. When $n \geq 2 \delta$,

$$
(n, \delta-3, \delta-4, \ldots, 1,0)>(2 \delta-1,2 \delta-3, \ldots, 7,5,4)
$$

in lexicographical order. When $n \leq 2 \delta-1$, we have the opposite comparison result. Therefore, the conclusion follows from Lemma 3.1

For each squarefree monomial $m=x_{i_{1}} \cdots x_{i_{k}} \in S$, we denote the set $\{1, \ldots, n\} \backslash$ $\left\{i_{1}, \ldots, i_{k}\right\}$ by $m^{\complement}$. Suppose $I$ is a squarefree monomial $S$-ideal and $G(I)$ is the set of minimal generating monomials of $I$. We call the simplicial complex $\Delta^{\complement}(I):=$ $\left\langle m^{\complement}: m \in G(I)\right\rangle$ the complement complex of $I$. For each simplicial complex over $[n]$, there is a unique squarefree monomial ideal $I$ such that $\Delta=\Delta^{\complement}(I)$. Thus, we will call $I$ the complement ideal of $\Delta$. It is clear that $I$ is generated by its degree $k$ part $I_{k}$ if and only if $\Delta^{\complement}(I)$ is pure of dimension $n-k-1$. When $\Delta^{\complement}(I)$ is pure, the number of facets $f_{n-k-1}\left(\Delta^{\complement}(I)\right)=\mu(I)$.

Now, let $I$ be a squarefree monomial ideal which is pure of degree $d$. We will relate the reduced associated poset $P_{I}^{d+1}$ of $I$ with its complement complex $\Delta^{\complement}(I)$. Each interval $[A, B] \subset P_{I}^{d+1}$ with $|A|=d$ and $|B|=d+1$ corresponds to the pair $([n] \backslash A,[n] \backslash B)$. Notice that $[n] \backslash A$ is a facet of $\Delta^{\complement}(I)$ and $[n] \backslash B$ is a face contained in $[n] \backslash A$. Now it is clear that the following three conditions are equivalent:

(a) The Stanley depth $\operatorname{sdepth}(I) \geq d+1$.

(b) The reduced associated poset $P_{I}^{d+1}$ is partitionable.

(c) For each facet $F$ of $\Delta=\Delta^{\complement}(I)$, we can suitably drop a vertex to get a face $\widetilde{F}$ such that all these $\widetilde{F}$ 's are pairwise distinct. 
The third condition is closely related to the problem of finding systems of distinct representatives (SDR). It provides the framework for our further investigation. In the following, we will call a pure simplicial complex $\Delta$ uniformly collapsible if it satisfies the third condition above. It is straightforward to see that if $\Delta$ is a uniformly collapsible complex of dimension $\delta-1$, then $f_{\delta-2} \geq f_{\delta-1}$. Here, $f(\Delta)=\left(f_{-1}=1, f_{0}, \ldots, f_{\delta-1}\right)$ is the $f$-vector of $\Delta$. Actually, we have the following characterization:

Lemma 3.3. For any $(\delta-1)$-dimensional pure simplicial complex $\Delta$, the following two conditions are equivalent:

(a) The complex $\Delta$ is uniformly collapsible.

(b) For each $(\delta-1)$-dimensional (pure) subcomplex $\Delta^{\prime}$, we have $f_{\delta-2}\left(\Delta^{\prime}\right) \geq f_{\delta-1}\left(\Delta^{\prime}\right)$.

Proof. For the pure complex $\Delta$, we consider its associated bipartite graph $G$ defined as follows. The vertex set is $V(G)=X \cup Y$, where $X$ is the set of all $(\delta-1)$ dimensional faces (facets) of $\Delta$, while $Y$ is the set of all $(\delta-2)$-dimensional faces of $\Delta$. An edge of $G$ has endpoints $x \in X$ and $y \in Y$ if and only if $x \supset y$ in $\Delta$. We will use $\Gamma(x)$ to denote the set of all vertices adjacent to a given vertex $x \in X$. If $A$ is a subset of $X$, we denote by $\Gamma(A)$ the set $\bigcup_{a \in A} \Gamma(a)$. Let $\Delta^{\prime}(A)$ be the simplicial complex $\langle A\rangle$. Then $\Gamma(A)$ is the set of all $(\delta-2)$-dimensional faces of $\Delta^{\prime}(A)$. Now, our claim follows directly from P. Hall's famous marriage theorem vLW01, 5.1], which says that a necessary and sufficient condition for there to be a complete matching from $X$ to $Y$ in $G$ is that $|\Gamma(A)| \geq|A|$ for every $A \subset X$. Since $f_{\delta-2}\left(\Delta^{\prime}(A)\right)=|\Gamma(A)|$ and $f_{\delta-1}\left(\Delta^{\prime}(A)\right)=|A|$, we are done.

Corollary 3.4. The pure simplicial complex $\Delta$ is uniformly collapsible if and only if $f_{\delta-2}(\Delta) \geq f_{\delta-1}(\Delta)$ and all its proper subcomplexes of the same dimension $\delta-1$ are uniformly collapsible.

Before we proceed to the next technical lemma, we need to review one nice combinatorial interpretation of the Catalan numbers $C_{n}:=\frac{1}{n+1}\left(\begin{array}{c}2 n \\ n\end{array}\right)=\left(\begin{array}{c}2 n \\ n\end{array}\right)-\left(\begin{array}{c}2 n \\ n+1\end{array}\right)$ for $n \geq 0$.

Remark 3.5 (vLW01, 14.8]). Consider walks in the $X-Y$ plane where each step is $U:(x, y) \rightarrow(x+1, y+1)$ or $D:(x, y) \rightarrow(x+1, y-1)$. Let $A=(0, k)$ and $B=(n, m)$ be two integral points on the upper halfplane. It follows from André's reflection principle that there are $\left(\begin{array}{l}n \\ l_{2}\end{array}\right)-\left(\begin{array}{l}n \\ l_{1}\end{array}\right)$ paths from $A$ to $B$ that do not meet the $X$-axis. Here, $2 l_{1}=n-k-m$ and $2 l_{2}=n-m+k$. As a result, there are $C_{n-1}$ paths from $(0,0)$ to $(2 n, 0)$ in the upper halfplane that do not meet the $X$-axis between these two points. Furthermore, if we allow the paths to meet the $X$-axis without crossing, then the number is $C_{n}$.

With respect to the Macaulay representation (10), we define

$$
\partial_{k-1}(x)=\left(\begin{array}{c}
a_{k} \\
k-1
\end{array}\right)+\left(\begin{array}{c}
a_{k-1} \\
k-2
\end{array}\right)+\cdots+\left(\begin{array}{c}
a_{i} \\
i-1
\end{array}\right) .
$$

Lemma 3.6. For any positive integer $x$ such that $x \leq \xi_{k}:=\sum_{j=1}^{k}\left(\begin{array}{c}2 j-1 \\ j\end{array}\right)$, we have $\partial_{k-1}(x) \geq x$.

Proof. Suppose (11) gives the Macaulay representation of $x$. We need to show

$$
\sum_{j=i}^{k}\left(\begin{array}{c}
a_{j} \\
j-1
\end{array}\right) \geq \sum_{j=i}^{k}\left(\begin{array}{c}
a_{j} \\
j
\end{array}\right) .
$$


In view of Lemma 3.1. we obtain $a_{k} \leq 2 k-1$. If $a_{k}=2 k-1$, we can consider the case where $k^{\prime}=k-1$ and $x^{\prime}=x-\left(\begin{array}{c}2 k-1 \\ k\end{array}\right)$. Now $x^{\prime} \leq \sum_{j=1}^{k^{\prime}-1}\left(\begin{array}{c}2 j-1 \\ j\end{array}\right)$. The conclusion will follow from the induction on $k$, with the case $k=1$ being trivial.

Thus we may assume that $a_{k}<2 k-1$. Let $k_{0}$ be the smallest integer such that for all $k_{0} \leq j \leq k$ we have $a_{j}<2 j-1$. Now, it suffices to prove

$$
\sum_{j=k_{0}}^{k}\left(\left(\begin{array}{c}
a_{j} \\
j-1
\end{array}\right)-\left(\begin{array}{c}
a_{j} \\
j
\end{array}\right)\right) \geq \sum_{j=i}^{k_{0}-1}\left(\left(\begin{array}{c}
a_{j} \\
j
\end{array}\right)-\left(\begin{array}{c}
a_{j} \\
j-1
\end{array}\right)\right) .
$$

First of all, let us look at the summand on the left hand side of inequality (2). By our choice of $k_{0}$, we have $k_{0}>1$ and $a_{k_{0}-1} \geq 2 k_{0}-3$. Thus, for $j=k_{0}, k_{0}+1, \ldots, k$, we have $a_{j} \geq j+k_{0}-2$. When $a_{j}<2 j-1$, the integer

$$
\left(\begin{array}{c}
a_{j} \\
j-1
\end{array}\right)-\left(\begin{array}{c}
a_{j} \\
j
\end{array}\right)=\left(\begin{array}{c}
a_{j} \\
a_{j}-j+1
\end{array}\right)-\left(\begin{array}{c}
a_{j} \\
a_{j}-j
\end{array}\right)
$$

is the number of paths in the $X-Y$ plane from $A=(0,1)$ to $B_{j, a_{j}}=\left(a_{j}, 2 j-1-a_{j}\right)$ that do not meet the $X$-axis. In particular, this is a positive integer. When $a_{j}<$ $2 j-2$, any such path followed by a step $D$ as in Remark 3.5 gives a path from $A$ to $B_{j, a_{j}+1}$. Thus, (3) is an increasing function for

$$
a_{j} \in\left\{j+k_{0}-2, j+k_{0}-1, \ldots, 2 j-2\right\}
$$

Now the infimum of the left hand side of (2) is achieved when $a_{j}=j+k_{0}-2$. Henceforth, without loss of generality, we may assume that $k=k_{0}$ and $a_{k}=2 k-2$, whence $a_{k-1}=2 k-3$.

Next, let us consider the summand on the right hand side of inequality (2). Notice that $a_{k}=2 k-2$; thus $a_{j} \leq k-2+j$. Now we have

$$
\left(\begin{array}{c}
a_{j} \\
j
\end{array}\right)-\left(\begin{array}{c}
a_{j} \\
j-1
\end{array}\right)=\left(\begin{array}{c}
a_{j} \\
j-1
\end{array}\right)\left(\frac{a_{j}-j+1}{j}-1\right)
$$

which is positive only when $a_{j} \geq 2 j-1$. When this condition is indeed satisfied, integer (4) is the number of paths in the $X-Y$ plane from $A=(0,1)$ to $B_{j, a_{j}}=$ $\left(a_{j}, a_{j}+1-2 j\right)$ that do not meet the $X$-axis. Any such path followed by a step $U$ as in Remark 3.5 gives a path from $A$ to $B_{j, a_{j}+1}$. Thus, (44) is an increasing function for $a_{j} \in\{2 j-1,2 j, \ldots, k-2+j\}$. Now the supremum of the right hand side of (2) is achieved when $i=1$ and $a_{j}=k-2+j$ for $j=1, \ldots, k-1$.

Now it suffices to prove

$$
\left(\begin{array}{c}
2 k-2 \\
k-1
\end{array}\right)-\left(\begin{array}{c}
2 k-2 \\
k
\end{array}\right) \geq \sum_{j=1}^{k-1}\left(\left(\begin{array}{c}
k-2+j \\
j
\end{array}\right)-\left(\begin{array}{c}
k-2+j \\
j-1
\end{array}\right)\right)
$$


As a matter of fact, we have

$$
\begin{aligned}
L H S-R H S= & \sum_{j=1}^{k}\left(\begin{array}{c}
k-2+j \\
j-1
\end{array}\right)-\sum_{j=1}^{k}\left(\begin{array}{c}
k-2+j \\
j
\end{array}\right) \\
= & \sum_{j=2}^{k}\left(\left(\begin{array}{c}
k-1+j \\
j-1
\end{array}\right)-\left(\begin{array}{c}
k-2+j \\
j-2
\end{array}\right)\right)+\left(\begin{array}{c}
k-1 \\
0
\end{array}\right) \\
& \quad-\sum_{j=1}^{k}\left(\left(\begin{array}{c}
k-1+j \\
j
\end{array}\right)-\left(\begin{array}{c}
k-2+j \\
j-1
\end{array}\right)\right) \\
= & \left(\left(\begin{array}{c}
2 k-1 \\
k-1
\end{array}\right)-\left(\begin{array}{c}
k \\
0
\end{array}\right)\right)+\left(\begin{array}{c}
k-1 \\
0
\end{array}\right)-\left(\left(\begin{array}{c}
2 k-1 \\
k
\end{array}\right)-\left(\begin{array}{c}
k-1 \\
0
\end{array}\right)\right) \\
= & 1 .
\end{aligned}
$$

One can also explain this difference being 1 by the paths argument in Remark 3.5 .

Next, consider the following property:

If $\Delta$ is a pure simplicial complex of dimension $\delta-1$ and $f_{\delta-1}(\Delta) \leq f_{\delta-2}(\Delta)$, then $\Delta$ is uniformly collapsible.

To investigate this property, we have to be equipped with further apparatus. We will need the following fact from Duv94, p79]. Define the reverse lexicographical order $\leq_{\text {rlex }}$ on the $k$-subsets of $[n]:=\{1,2, \ldots, n\}$ as follows. Let $S=\left\{i_{1}<\cdots<i_{k}\right\}$ and $T=\left\{j_{1}<\cdots<j_{k}\right\}$ be two $k$-subsets. We say $S<_{\text {rlex }} T$ if for some $q$, we have $i_{q}<j_{q}$ and $i_{p}=j_{p}$ for $p>q$. A collection $C$ of $k$-subsets of $[n]$ is compressed if $S<_{\text {rlex }} T$ and $T \in C$ imply $S \in C$. Since $\leq_{\text {rlex }}$ is a total ordering, there is only one compressed collection of $k$-subsets of size $l, 1 \leq l \leq\left(\begin{array}{l}n \\ k\end{array}\right)$. We will call it $C_{n, k}^{l}$ and denote the $(k-1)$-dimensional simplicial complex $\left\langle C_{n, k}^{l}\right\rangle$ by $\Delta_{l}^{n, k}$. The complement ideal of $\Delta_{l}^{n, k}$ will be written as $I_{n, n-k}^{l}$. It is generated by $l$ squarefree monomials of degree $n-k$. For $1 \leq d \leq n$ and $l=\left(\begin{array}{l}n \\ d\end{array}\right)$, the ideal $I_{n, d}^{l}$ is the usual squarefree Veronese ideal $I_{n, d}$.

The shadow of any collection $C$ of $k$-subsets is

$$
\partial C=\{S:|S|=k-1, S \subset T \text { for some } T \in C\} .
$$

The shadow $\partial C_{n, k}^{l}$ is also compressed and $\left|\partial C_{n, k}^{l}\right|=\partial_{k-1}(l)$. The proof of this fact can be found, for instance, in [GK78, Section 8]. This implies that $f_{k-2}\left(\Delta_{l}^{n, k}\right)=$ $\partial_{k-1}\left(f_{k-1}\left(\Delta_{l}^{n, k}\right)\right)=\partial_{k-1}(l)$.

When $\Delta$ is pure of dimension $\delta-1$ and $C$ is the set of all facets, then $\partial C$ is the set of all $(\delta-2)$ faces. In general, we will have $f_{\delta-2}(\Delta) \geq \partial_{\delta-1}\left(f_{\delta-1}(\Delta)\right)$, namely $|\partial C| \geq \partial_{\delta-1}(|C|)$; see [GK78, 8.1].

Example 3.7. The simplicial complex $\Delta=\Delta_{\xi_{\delta}+1}^{n, \delta}$ is not uniformly collapsible. For this, it suffices to observe that $f_{\delta-1}(\Delta)=\xi_{\delta}+1=\left(\begin{array}{l}2 \\ 1\end{array}\right)+\sum_{j=2}^{\delta}\left(\begin{array}{c}2 j-1 \\ j\end{array}\right)$. Thus $f_{\delta-2}(\Delta)=\partial_{\delta-1}\left(\xi_{\delta}+1\right)=\left(\begin{array}{l}2 \\ 0\end{array}\right)+\sum_{j=2}^{\delta}\left(\begin{array}{c}2 j-1 \\ j-1\end{array}\right)=\xi_{\delta}$ and $f_{\delta-1}(\Delta)>f_{\delta-2}(\Delta)$. Now apply Corollary 3.4 . 
If we combine Corollary 3.4 with Lemma 3.6, we obtain the following result:

Corollary 3.8. If $f_{\delta-1}(\Delta) \leq \xi_{\delta}$, then property $(*)$ holds.

However, property $(*)$ does not hold in general.

Example 3.9. We already know that the simplicial complex $\Delta_{\xi_{\delta}+1}^{n, \delta}$ over the vertex set $[n]$ is not uniformly collapsible. Now, let $\widetilde{\Delta}=\left\langle\Delta_{\xi_{\delta}+1}^{n, \delta},\{n, n+1, \ldots, n+\delta-1\}\right\rangle$ be a new simplicial complex over the vertex set $[n+\delta-1]$. It is again pure of dimension $\delta-1$. Notice that

$$
f_{\delta-1}(\widetilde{\Delta})=f_{\delta-1}(\Delta)+1=\xi_{\delta}+2
$$

and, when $\delta \geq 3$,

$$
f_{\delta-2}(\widetilde{\Delta})=f_{\delta-2}\left(\Delta_{\xi_{\delta}+1}^{n, \delta}\right)+f_{\delta-2}(\langle\{n, n+1, \ldots, n+\delta-1\}\rangle)=\xi_{\delta}+\delta .
$$

Hence, we have $f_{\delta-2}(\widetilde{\Delta})>f_{\delta-1}(\widetilde{\Delta})$. However, $\widetilde{\Delta}$ is not uniformly collapsible because of the existence of the pure subcomplex $\Delta_{\xi_{\delta}+1}^{n, \delta}$.

In the current context, we always assume that $n / 2 \leq d \leq n$, whence $2 \delta \leq n$. The obstacle in the previous example is created by introducing extra vertices; now the number of vertices is at least $3 \delta-1$. Thus, we are interested in the following question:

Question 3.10. Fix the degree difference $\delta$. If $n=2 \delta$, does property $(*)$ hold? If the answer is positive, what is the largest integer $n<3 \delta-1$ such that (*) holds?

\section{Proof of Theorem 1.1}

We have gathered all the apparatus for proving the main theorem.

Proof. By virtue of Remark 2.2 we may assume that $I$ is pure of degree $d$. For $1 \leq d<n$, write $\delta=n-d$ for the difference of degrees.

When $n \geq 2 d+1$, we have $n \leq 2 \delta-1$. Thus

$$
\min \left(\xi_{n-d},\left(\begin{array}{c}
n \\
d+1
\end{array}\right)\right)=\left(\begin{array}{c}
n \\
d+1
\end{array}\right)
$$

by virtue of Lemma 3.2 The condition $\mu(I) \leq\left(\begin{array}{c}n \\ d+1\end{array}\right)$ is automatically satisfied, and we have $\operatorname{sdepth}(I) \geq d+1$ from Proposition 2.5 .

On the other hand, when $1 \leq d<n \leq 2 d$, we have $n \geq 2 \delta$. Now

$$
\min \left(\xi_{n-d},\left(\begin{array}{c}
n \\
d+1
\end{array}\right)\right)=\xi_{n-d}
$$

If $\mu(I) \leq \xi_{\delta}$, its complement complex $\Delta^{\complement}(I)$ is uniformly collapsible from Lemmas 3.3 and 3.6. Thus $\operatorname{sdepth}_{S}(I) \geq d+1$.

Remark 4.1. We want to emphasize that the condition in Theorem 1.1 is optimal. With $\delta=n-d$, there is not much to mention for the case $n \leq 2 \delta-1$. When $n \geq 2 \delta$, we will take $I=I_{n, d}^{\xi_{\delta}+1}$. It has been manifested in Example 3.7 that the complement complex $\Delta_{\xi_{\delta}+1}^{n, \delta}$ is not uniformly collapsible, whence $\operatorname{sdepth}_{S}(I)=d$. We finish by noticing that $\mu_{d}(I)=\xi_{\delta}+1$. 
Remark 4.2. When $n / 2 \leq d<n$, the set

$$
\Xi:=\{I \subset S \mid I \text { is pure of degree } d \text { and } \operatorname{sdepth}(I)=d\}
$$

is nonempty and partially ordered with respect to inclusion. If $I \in \Xi$ is minimal, then $\mu(I) \geq \xi_{\delta}+1$. This inequality can be strict if the dimension $n$ is not too small relative to the difference $\delta=n-d$. We will only show this in the special case when $d=n-2$. Let $G$ be the graph on $[n]$ (1-dimensional pure simplicial complex) with edges

$$
E(G)=\{\{1,2\},\{2,3\}, \ldots,\{n-1, n\},\{n, 1\},\{1,3\}\} .
$$

It is a circle with a chord. All 1-dimensional proper subcomplexes of $G$ are uniformly collapsible, while $G$ itself is not. Let $I$ be the degree $n-2$ complement ideal of the complex $G$. It satisfies that $\operatorname{sdepth}(I)=n-2$ and $\mu(I)=n+1$. Furthermore, this ideal is minimal in $\Xi$.

Since $n+1$ is smaller when compared with $\left(\begin{array}{l}n \\ d\end{array}\right)$ or $\left(\begin{array}{c}n \\ d+1\end{array}\right)$ in this situation, we are interested in

Question 4.3. What is $\max \{\mu(I) \mid I$ is minimal in $\Xi\}$ ?

Since $\left(\begin{array}{l}n \\ d\end{array}\right)>\left(\begin{array}{c}n \\ d+1\end{array}\right)$, the maximal element of $\Xi$ is the squarefree Veronese ideal $I_{n, d}$. Thus, the number

$$
\max \{\mu(I) \mid I \text { is maximal in } \Xi\}
$$

is clear.

Remark 4.4. When $n / 2 \leq d<n$, the set

$$
\Xi^{\complement}:=\{I \subset S \mid I \text { is pure of degree } d \text { and } \operatorname{sdepth}(I)>d\}
$$

is also nonempty. For any $I \in \Xi^{\mathrm{C}}$, we have $\mu(I) \leq\left(\begin{array}{c}n \\ d+1\end{array}\right)$. We will show that

$$
\max \left\{\mu(I) \mid I \in \Xi^{\mathrm{C}}\right\}=\left(\begin{array}{c}
n \\
d+1
\end{array}\right) .
$$

Suppose $k$ is an integer with $1 \leq k \leq n-1$. If a squarefree monomial ideal $I$ is pure of degree $k$ and $\operatorname{sdepth}(I) \geq k+1$, we have a union of disjoint intervals $\bigcup_{\mathbf{x}^{m} \in G(I)}[m, \widetilde{m}]$ in $P_{I}^{k+1}$, with $|\widetilde{m}|=k+1$ for each $\mathbf{x}^{m} \in G(I)$. Here, $\mathbf{x}^{m}$ stands for $x_{i_{1}} x_{i_{2}} \cdots x_{i_{k}}$ if $m=\left\{i_{1}, \ldots, i_{k}\right\}$. Now, simply set $J=\left\langle\mathbf{x}^{\widetilde{m}^{\mathrm{C}}} \mid m \in G(I)\right\rangle$. The squarefree monomial ideal $J$ is pure of degree $n-k-1$ and $\operatorname{sdepth}(J) \geq n-k$. This correspondence from $I$ to $J$, though not one-to-one, preserves the minimal number of generators.

Now, we are reduced to show the existence of a squarefree monomial ideal $J$ that is pure of degree $n-d-1$ with $\mu(J)=\left(\begin{array}{c}n \\ d+1\end{array}\right)$ and $\operatorname{sdepth}(J) \geq n-d$. This monomial ideal $J$ has to be the squarefree Veronese ideal $I_{n, n-d-1}$. Since $2 d \geq n-1$, it has the desired properties.

Note that any set of squarefree monomials has a squarefree shadow; see BEOS09, $2.2]$. Thus, we can prove Theorem 1.1 directly without resorting to the complement complex. However, we find this approach less intuitive, especially during the construction of the simplicial complex $\widetilde{\Delta}$ in Example 3.9 and the graph $G$ in Remark 4.2 


\section{REFERENCES}

[BEOS09] V. Bonanzinga, V. Ene, A. Olteanu, and L. Sorrenti, An overview on the minimal free resolutions of lexsegment ideals, Combinatorial aspects of commutative algebra, Contemp. Math., vol. 502, Amer. Math. Soc., Providence, RI, 2009, pp. 5-24. MR2583270 (2011e:13025)

[BH93] W. Bruns and J. Herzog, Cohen-Macaulay rings, Cambridge Studies in Advanced Mathematics, vol. 39, Cambridge University Press, Cambridge, 1993 . MR 1251956 (95h:13020)

$\left[\mathrm{BHK}^{+} 10\right]$ C. Biró, D. M. Howard, M. T. Keller, W. T. Trotter, and S. J. Young, Interval partitions and Stanley depth, J. Combin. Theory Ser. A 117 (2010), no. 4, 475-482. MR2592896 (2011e:13040)

[BKU10] W. Bruns, C. Krattenthaler, and J. Uliczka, Stanley decompositions and Hilbert depth in the Koszul complex, J. Commut. Algebra 2 (2010), 327-357. MR.2728147 (2011j:13027)

[Cim09] M. Cimpoeaş, Stanley depth of square free Veronese ideals (2009), available at arXiv: 0907.1232.

[Duv94] A. M. Duval, A combinatorial decomposition of simplicial complexes, Israel J. Math. 87 (1994), no. 1-3, 77-87. MR1286816 (96e:52023)

[Gar80] A. M. Garsia, Combinatorial methods in the theory of Cohen-Macaulay rings, Adv. in Math. 38 (1980), no. 3, 229-266. MR597728 (82f:06002)

[GK78] C. Greene and D. J. Kleitman, Proof techniques in the theory of finite sets, Studies in Combinatorics, MAA Stud. Math., vol. 17, Math. Assoc. America, Washington, D.C., 1978, pp. 22-79. MR513002 (80a:05006)

[HJY08] J. Herzog, A. S. Jahan, and S. Yassemi, Stanley decompositions and partitionable simplicial complexes, J. Algebraic Combin. 27 (2008), no. 1, 113-125. MR2366164 (2008m:13041)

[HVZ09] J. Herzog, M. Vladoiu, and X. Zheng, How to compute the Stanley depth of a monomial ideal, J. Algebra 322 (2009), 3151-3169. MR2567414 (2010k:13036)

[KSSY11] M. T. Keller, Y.-H. Shen, N. Streib, and S. J. Young, On the Stanley depth of squarefree Veronese ideals, J. Algebraic Combin. 33 (2011), no. 2, 313-324. MR2765327 (2012d:13033)

[KY09] M. T. Keller and S. J. Young, Stanley depth of squarefree monomial ideals, J. Algebra 322 (2009), no. 10, 3789-3792. MR2568363 (2010h:13018)

[Oka11] R. Okazaki, A lower bound of Stanley depth of monomial ideals, J. Commut. Algebra 3 (2011), no. 1, 83-88. MR2782700 (2012d:13036)

[She09] Y. H. Shen, Stanley depth of complete intersection monomial ideals and upper-discrete partitions, J. Algebra 321 (2009), no. 4, 1285-1292. MR2489900 (2009k:13044)

[Sta79] R. P. Stanley, Balanced Cohen-Macaulay complexes, Trans. Amer. Math. Soc. 249 (1979), no. 1, 139-157. MR526314 (81c:05012)

[Sta82] Linear Diophantine equations and local cohomology, Invent. Math. 68 (1982), no. 2, 175-193. MR666158 (83m:10017)

[vLW01] J. H. van Lint and R. M. Wilson, A course in combinatorics, 2nd ed., Cambridge University Press, Cambridge, 2001. MR1871828 (2002i:05001)

The Wu Wen-Tsun Key Laboratory of Mathematics of CAS and School of Mathematical Sciences, University of Science and Technology of China, Hefei, Anhui, 230026 , People's Republic of China

E-mail address: yhshen@ustc.edu.cn 